Service social

\title{
Réflexions sur les techno-sciences et l'instrumentalisation dans la procréation humaine
}

\section{Maria De Koninck et Marie-Hélène Parizeau}

Volume 40, numéro 1, 1991

Éthique et intervention sociale

URI : https://id.erudit.org/iderudit/706512ar

DOI : https://doi.org/10.7202/706512ar

Aller au sommaire du numéro

Éditeur(s)

École de service social de l'Université Laval

ISSN

1708-1734 (numérique)

Découvrir la revue

Citer cet article

De Koninck, M. \& Parizeau, M.-H. (1991). Réflexions sur les techno-sciences et l'instrumentalisation dans la procréation humaine. Service social, 40(1), 11-30. https://doi.org/10.7202/706512ar
Résumé de l'article

Les auteures situent globalement la problématique des techno-sciences pour ensuite s'attarder aux aspects critiques de celles-ci dans le champ de la procréation humaine. Elles poursuivent en décrivant certaines conséquences de l'utilisation de la norme du risque génétique pour le développement des techno-sciences et insistent sur l'instrumentalisation du corps qui en résulte. Enfin, elles proposent des valeurs qui pourraient fonder les choix sociaux dans le domaine et formulent des recommandations dont la mise en oeuvre favoriserait la définition d'une approche globale des effets et des conséquences du développement des techniques de procréation. 


\title{
ARTICLES
}

Maria De Koninck, titulaire de la chaire d'étude sur la condition des femmes et professeure, Département de médecine sociale et préventive, Université Laval.

Marie-Hélène Parizeau, professeure, Faculté de philosophie, Université Laval.

\section{Réflexions sur les techno-sciences et l'instrumentalisation dans la procréation humaine}

\author{
Maria De Koninck \\ Marie-Hélène Parizeau
}

\section{Introduction}

Les nouvelles techniques de procréation humaine développées par la médecine, de l'insémination artificielle à la fécondation in vitro en passant par le diagnostic prénatal, ont été amenées très rapidement à la connaissance du grand public grâce à leur côté spectaculaire et a priori réjouissant (faire naître un bébé). Plus de dix ans après la naissance de Louise Brown, première enfant issue de la technique de la fécondation in vitro, les questions sur le développement de ces techniques, leur accessibilité, leur financement, leurs implications éthiques se posent avec de plus en plus d'acuité. Plusieurs pays ont déjà amorcé des réflexions en ce sens : en Angleterre, la commission 
Warnock en 1984, en Allemagne, la commission Benda en 1985, en France, le Comité consultatif national d'éthique en 1986, etc. Au Canada, la Commission de réforme du droit de l'Ontario a fourni le premier rapport en 1985. Au Québec, ce sont des experts réunis en comité qui ont formulé des recommandations : le comité du ministère de la Santé et des Services sociaux en 1988 et le comité du Barreau du Québec la même année. Ces différents efforts de réflexion ainsi que la pression exercée par divers groupes à travers le Canada ont abouti à la création sur le plan politique d'une Commission royale d'enquête sur les nouvelles techniques de la reproduction en 1989.

Le texte qui suit a été soumis à la Commission. Notre participation à ses travaux tient à des préoccupations tant professionnelles que personnelles concernant la santé reproductive des femmes et l'éthique sociale. Ces préoccupations nous ont menées à des interrogations sur les choix effectués actuellement dans le domaine des technologies de procréation et sur leurs conséquences à court et à long terme pour notre société.

Dans un premier temps nous situons globalement la problématique des techno-sciences pour ensuite nous attarder aux aspects critiques des techno-sciences dans le champ de la procréation. Puis, nous insistons sur l'instrumentalisation du corps par la technique pour ensuite prendre position sur certaines valeurs qui nous paraissent devoir fonder les choix sociaux. Enfin, nous formulons des recommandations afin de développer une approche globale concernant les effets et conséquences du développement des techniques de procréation.

\section{Le recours à la technique et son application à l'humain}

L'utilisation de la technique est intrinsèquement liée au devenir de l'être humain, de la pierre taillée à l'invention de la roue, de l'invention de l'imprimerie à celle de l'ordinateur. On pourrait dresser la même trajectoire dans le domaine de la médecine, de l'utilisation des plantes médicinales aux trépanations effectuées par les médecins des dynasties égyptiennes, à l'invention du stéthoscope, en passant par la ventilation artificielle et la cartographie génétique.

Si la technologie ancienne s'est développée lentement et sur les bases d'un savoir-faire pratique, sans véritable justification théorique, il en va tout autrement des technologies modernes. La technologie moderne est intimement liée au développement de la science, au point que les deux forment un couple : les techno-sciences. Par les modèles théoriques, la science fournit un certain savoir sur la réalité. 
Par sa méthode, elle rend possible un accroissement réglé de ce savoir en l'améliorant. La démarche scientifique permet le progrès, c'est-à-dire un certain type d'évolution. Ce savoir sur les choses, de type opératoire, constitue par le fait même un pouvoir sur les choses et sur l'humain. C'est la technologie qui concrétise ce pouvoir par la transformation du réel. " En bref, dit Ladrière, on pourrait dire que la science a pour objectif le progrès de la connaissance, alors que la technologie a pour objectif la transformation de la réalité donnée » (Ladrière, $1979: 57$ ). L'être humain acquiert ainsi des outils technoscientifiques qui lui permettent de modifier le cours des lois de la nature (par exemple, la sélection naturelle). Or, modifier le cours naturel d'un phénomène (telle une maladie) veut dire contrôler les conditions de l'évolution de ce phénomène afin d'intervenir par divers moyens techniques pour en changer le déroulement. Les technosciences demandent, d'une part, une connaissance sur le phénomène (son déroulement, ses causes) et, d'autre part, des moyens de contrôler son développement afin de le modifier. En résumé, les technosciences sont un outil de transformation du réel.

Les techno-sciences évoluent rapidement et ont un caractère de plus en plus systématique, donc de plus en plus contrôlé. Elles se présentent socialement comme des recherches organisées et couplées à un mode d'organisation de la production de l'industrie moderne où l'on retrouve une très grande division du travail, un haut niveau d'intégration des activités et une production de masse. Sur le plan critique, les techno-sciences répondent à un impératif d'utilité; elles sont fonctionnelles, c'est-à-dire au service d'autres activités. En cela, elles consistent en " un ensemble de moyens sans finalités propres» (Freund, $1983: 24$ ). Les techno-sciences, qui n'ont pas de fin propre, possèdent néanmoins des objectifs concrets, ponctuels, régulés par les lois de l'efficacité et du rendement. On est alors dans l'ordre du pragmatique. Mais les techno-sciences ne sont pas pour autant des outils neutres, car l'utilisation de ces outils induit des conséquences sur le plan des comportements humains et de la psychologie des individus qui s'en servent. Les techno-sciences ont des orientations, des implications et des conditions de fonctionnement et en cela modifient l'expérience humaine et son environnement. Les techno-sciences deviennent la réalité et chaque être humain doit s'adapter psychologiquement et comportementalement aux technologies qui l'entourent (le téléphone, la télévision, l'ordinateur, etc.). La plus parfaite adaptation à une technique particulière permet de tirer le maximum de profit de la technique vers d'autres finalités. Bref, les techno-sciences modèlent les comportements humains et médiatisent le rapport au réel.

Aussi, sur le plan éthique, les techno-sciences sont-elles devenues en soi une valeur. Le progrès techno-scientifique est devenu 
porteur du bien futur et du bonheur individuel. On peut parler d'une " éthique technicienne » (Ellul, $1983: 13$ ) à partir de laquelle on juge les comportements dans notre société. II n'y a pas d'échelle de valeurs fruit $d^{\prime}$ 'une construction intellectuelle, mais on substitue aux normes éthiques les modes de fonctionnement des techno-sciences : normalité, efficacité, réussite, travail, conscience professionnelle, dévouement à l'œuvre collective, etc. Avec comme corollaire le rejet d'autres conduites : gratuité, inefficacité, perte de temps, bonté, humour, fidélité, etc. Les modes de fonctionnement des technosciences permettent une meilleure adaptation de l'être humain à son environnement techno-scientifique (machines, procédés, etc.). L'adaptation est renforcée par des incitations à la production, à la consommation. L'inadapté social est celui qui, par exemple, ne sait pas ou ne veut pas se servir d'un ordinateur dans le cadre de son travail ou encore, sur le plan médical, c'est la femme enceinte qui refuse les "bienfaits " de l'accouchement sans douleur par péridurale.

Ayant ainsi situé globalement les implications des technosciences comme moyens de contrôle sur un événement donné, c'està-dire aussi sur le réel et sur l'être humain, on saisit mieux l'importance d'une réflexion critique sur les développements technoscientifiques et la nécessité d'une interrogation éthique.

\section{Les techno-sciences et la procréation humaine : aspects critiques}

\section{Connaissances et normes techno-scientifiques}

Les techno-sciences qui se sont développées autour de la grossesse et de la procréation humaine procèdent d'une part par l'acquisition de connaissances sur des phénomènes de la grossesse (de la fécondation au fœetus) qui ont ou non des conséquences mortelles ou débilitantes pour le fœtus et, d'autre part, par la mise en œuvre de contrôles plus ou moins efficaces en vue de modifier le processus naturel ou pathologique de la grossesse.

Par exemple, la technique de l'échographie permet de détecter les cas où des fœtus ont certaines malformations cardiaques qui peuvent être corrigées à la naissance; mais le fait même de ce diagnostic précoce entraînera des conditions spéciales de surveillance de la grossesse et surtout de l'accouchement. Ici, c'est tout le processus de la grossesse et de l'accouchement qui est modifié et contrôlé à partir des informations de l'échographie. Les normes techno-scientifiques adoptées socialement, auxquelles chacun réitère son adhé- 
sion - l'équipe soignante, la femme enceinte et le conjoint - font qu'il faut sauver cet enfant, puisque les moyens techniques et médicaux existent et que chacun veut le bien de l'enfant à naître. D'autant plus que la technique peut prédire avec un certain degré de certitude que les chances de réussite de l'opération et de guérison sont très élevées. Les modes de fonctionnement des techno-sciences - normalité, efficacité, réussite, travail, conscience professionnelle, dévouement à l'œuvre collective - s'allient en somme à la finalité éthique qui est de sauver l'enfant à naître.

Cependant, les normes techno-scientifiques n'offrent plus les mêmes convergences vers une même finalité éthique lorsque, à la suite d'une amniocentèse, la femme (le couple) apprend qu'elle porte un enfant trisomique. La femme (le couple) aura à choisir si elle continue la grossesse ou y met un terme. Le discours médical sur le phénomène de la trisomie renvoie généralement à un jugement d'anormalité du fœtus porteur d'une trisomie 21. Les pressions sociales vont également dans ce sens : un enfant trisomique n'est pas un enfant comme les autres; à cause de son handicap mental il serait de qualité moindre. La détermination de la finalité éthique est alors renvoyée à la femme (au couple), puisque la technique n'offre pas de réponses techniques satisfaisantes (il n'y a pas moyen de corriger le handicap). La décision devient alors de l'ordre d'une responsabilité individuelle, et non plus collective, que doit assumer tragiquement et isolément la femme (le couple).

Les techno-sciences, dans le cadre de la grossesse et de la procréation humaine, sont porteuses de contrôles sur les phénomènes, mais elles sont également porteuses de normes éthiques. En matière de procréation humaine, ces normes ont pour objet le normal et l'anormal, la qualité et la défectuosité, la perfection et l'imperfection, la responsabilité et l'irresponsabilité. Autant de dichotomies et d'antagonismes à partir desquels les techno-sciences déterminent leurs modes d'intervention.

On peut s'interroger sérieusement sur la légitimité de ces normes (qui définit les normes ? suivant quelles modalités?). En tout cas, on peut s'interroger sur les choix de valeurs sous-jacents et réfléchir sur l'idéal qui est véhiculé par les techno-sciences. Nous ouvrirons cette discussion un peu plus loin.

\section{Une norme techno-scientifique particulièrement efficace : le risque génétique}

Le continuel développement de techno-sciences en matière de procréation humaine s'appuie tout particulièrement sur une norme 
simple et efficace : le risque génétique. Les techno-sciences procèdent par contrôles des phénomènes, nous l'avons dit. Aussi les contrôles techno-scientifiques, dès qu'ils sont dans l'ordre du possible, vont-ils se multiplier à partir du moment où sont mesurées techniquement des modifications qui vont perturber le développement connu du phénomène - ici, le développement du fœtus. Bref, la connaissance et la mesure du risque par la technique entraînent quasi automatiquement l'intervention techno-scientifique.

Nous sommes tous porteurs de gènes délétères et leur combinaison au moment de la fécondation et dans le développement ultérieur du fœetus peut entraver le développement de celui-ci, affectant de façon plus ou moins importante sa viabilité. Or, la norme technoscientifique du risque génétique amplifie le facteur $\mathrm{d}^{\prime}$ 'incertitude lié à l'apparition de toute nouvelle existence humaine et intervient sur le plan des comportements humains.

Les psychologues et psychanalystes ont amplement exploré les ambivalences de l'attente et du désir d'enfant : espérance et peur de l'inconnu, enfant idéalisé et enfant réel, projection de soi et altérité radicale. La norme techno-scientifique du risque génétique vient jouer, sur le plan psychologique, sur le registre de la peur de l'inconnu, sur l'enfant idéalisé, voire l'enfant parfait, et sur la projection de soi dans l'autre.

Les techno-sciences (du diagnostic prénatal à la fécondation in vitro), par les contrôles qu'elles proposent dans le déroulement de la grossesse, donnent des moyens d'agir sur le devenir et sur le développement de l'embryon comme du fœtus de façon plus ou moins limitée suivant la pathologie, en tout cas de plus en plus précise en matière de détection de maladies génétiques et de prédispositions aux maladies.

Le fantasme du façonnement de l'autre à son image, qui fait partie comme d'autres fantasmes de toute expérience de la maternité, est singulièrement renforcé et surtout actualisé par les techno-sciences. L'identification du sexe du fœtus par amniocentèse à un stade assez précoce, comme cela se pratique en Inde par exemple, permet de réaliser le fantasme (psychologique et issu de pressions sociales) du garçon premier-né, en éliminant le cas échéant le fœtus de sexe féminin par avortement (une autre intervention technique). La technique permet ici la standardisation du produit (le garçon premier-né) à grande échelle.

En bref, par la connaissance que l'on acquiert sur le fœtus ou l'embryon, les techno-sciences permettent, par les moyens d'intervention offerts, de définir l'Autre en termes de normal ou d'anormal, d'acceptable ou d'inacceptable. Les techno-sciences deviennent ainsi 
volonté de puissance sur le réel qui est, a priori, sans limite intrinsèque.

Les techno-sciences entraînent également le processus $d^{\prime}$ objectivation du réel. Le fœetus et l'embryon sont qualifiés et définis en des termes mesurables. Ceux-ci sont d'emblée réducteurs de la totalité du réel, puisqu'ils participent à la réalité scientifique (expérimentale, transformatrice, objectivante, prédictive). Le discours éthique est complètement éliminé pour n'être réintroduit que sur le plan de la finalité de l'objet qu'est devenu le fœtus ou l'embryon. Le processus d'objectivation permet éventuellement de changer le cours du développement du fœtus par le contrôle des facteurs jugés délétères dont le fœtus serait porteur.

Le phénomène d'objectivation des techno-sciences a tout particulièrement des répercussions sur l'ordre de la connaissance de la maternité. Il impose la norme du savoir et invalide les autres formes de connaissances, en particulier la connaissance vécue individuelle (l'écoute du corps). Si, par exemple, certaines femmes qui se présentent à l'hôpital au moment de l'accouchement désirent vivre le plus possible leur accouchement et accoucher " en acte " avec le minimum d'interventions médicales, d'autres femmes viennent "se faire " accoucher par la technique (demande de péridurale ou de césarienne). La technique permet le retrait et le détachement de l'expérience relationnelle, ici de l'accouchement de son propre enfant. Elle permet d'être à l'extérieur de l'événement, comme observatrice de soi-même, tel le scientifique objectif qui observe le phénomène physiologique. Les techno-sciences induisent donc des pressions, voire des déformations, dans l'expérience de la maternité.

Enfin, le phénomène d'objectivation mesure la maternité, non pas comme une expérience vécue, mais en termes de produit : I'enfant né ou à naître. Ce produit de la maternité est qualifiable (utilité, qualité, degré de perfection, etc.) par les contrôles techniques : ce fœtus ou ce bébé est-il en santé ? Présente-t-il des anormalités ? Les techno-sciences peuvent ainsi développer des critères de qualification du produit, et le glissement de l'enfant en santé à l'enfant parfait, par la mise en œuvre de contrôles génétiques par exemple, ne devient qu'une modalité techno-scientifique de mesure de qualité.

La conséquence ultime de la norme du risque génétique et du discours qui y est associé, c'est un refus de l'anormalité, la peur de la différence et le renforcement de l'élément narcissique du couple et de la femme, c'est-à-dire une perception de l'Autre comme une continuité du Moi, voire une projection du Moi, donc ultimement le refus de l'Altérité. Ainsi brièvement se définit l'instrumentalisation de l'autre par la techno-science, avec comme corollaire la dramatisation croissante de l'anormalité, du handicap. 


\section{L'instrumentalisation du corps par la technique}

\section{La progression historique du recours à la technique}

Dans la mesure où l'on veut contrôler le "produit de la procréation humaine ", on cherche à étendre ce contrôle au processus luimême, d'où la justification d'une emprise croissante sur le corps féminin reproducteur, de sorte que les développements techniques ont comme effet de transformer l'expérience des femmes. C'est en situant ces développements dans une perspective historique qu'on peut saisir la logique sous-jacente à l'évolution technologique qui a provoqué cette transformation.

Le recours à des instruments dans le processus de reproduction est relativement récent, l'usage des forceps, première véritable incursion technique dans la procréation, ne s'étant en effet répandu qu'au dix-neuvième siècle. Les conséquences de l'introduction et de la diffusion de cet instrument au moment de l'accouchement témoignent du potentiel d'impact social d'une telle "nouveauté ». Son usage a notamment contribué au développement de l'obstétrique et à la redéfinition du rôle des sages-femmes, quand ce n'est pas à leur éviction comme ce fut le cas en Amérique du Nord (Wertz, Wertz, 1979).

La diffusion des forceps s'inscrivait dans un changement de mentalité qui correspondait à une nouvelle vision mécaniste de l'univers. $C^{\prime}$ est en effet cette vision globale qui sous-tend la perception du corps humain comme étant une machine susceptible de se briser. Appliquée au corps féminin reproducteur et au processus de procréation cette perception a favorisé la multiplication des interventions techniques. Ces dernières se sont largement développées depuis, soutenues par une évolution de la vision de l'univers selon laquelle on arrive aujourd'hui à percevoir le corps comme un système à maintenir sous surveillance (Arney, 1982).

Cette évolution est également perceptible dans la progression de la technique. Celle-ci s'est accompagnée d'une remise en question de la définition de la contribution des femmes à la procréation. Déjà, en 1920, le Dr Joseph De Lee, autorité en obstétrique, préconisait l'utilisation routinière des forceps, sous prétexte que l'expulsion naturelle de l'enfant pouvait provoquer des traumatismes crâniens'.

La multiplication des interventions et leur normalisation se sont aussi accompagnées d'un élargissement des lieux d'intervention. En effet, aux interventions au moment de l'accouchement s'en sont progressivement ajoutées d'autres au cours de la grossesse (Oakley, 1984) et finalement dans la conception.

Dans une perspective historique, l'évolution est donc récente et très rapide. Le moteur de cette évolution est la recherche d'un meil- 
leur contrôle sur le processus de procréation. Or, le siège de ce processus est le corps féminin; de là, une emprise croissante sur le corps féminin reproducteur. Cette emprise se traduit non seulement par des interventions au moment de l'accouchement, mais également par une surveillance et un contrôle du corps avant l'enfantement : les comportements des femmes enceintes sont normés et la technique est utilisée pour sanctionner le déroulement de la grossesse selon les règles et prescriptions médicales.

II importe donc, pour comprendre les enjeux et dégager la logique des développements techniques, de garder à l'esprit que la situation actuelle est une étape dans un processus amorcé depuis déjà un certain temps. Mettre les développements dans une telle perspective favorise la réflexion sur les raisons d'être des nouvelles pratiques.

Ces raisons se sont progressivement déplacées d'une préoccupation axée sur la santé de la mère vers une préoccupation axée sur la qualité du produit (le fœtus). L'évolution s'est aussi accompagnée de changements dans les standards de production (poids de l'enfant, absence de handicap et même forme du crâne...) où l'on vise une performance accrue.

Les conséquences de cette évolution sont multiples. Toutefois, deux aspects particuliers méritent d'être soulignés ici, la réification de l'expérience de la procréation et la déqualification du savoir des femmes.

\section{La réification de l'expérience de la procréation}

L'expérience de la procréation est une expérience humaine globale. Elle sollicite le corps bien sûr, mais aussi l'esprit, et s'inscrit dans une réalité historique et sociale. Or, la possibilité de concrétiser les standards de production souhaités aujourd'hui exige que l'on puisse procéder à des manipulations dans le processus de la procréation. Seules les dimensions physiques et physiologiques de l'expérience sont manipulables, elles deviennent alors dominantes. Ce qui ne peut être traité techniquement sort progressivement du champ du réel; ainsi les dimensions de l'expérience autres que physiques et physiologiques n'ont plus d'existence.

Cette donnée est importante dans une réflexion sur les conséquences de la progression technique dans le domaine. L'expérience des femmes au moment de la grossesse, par exemple, n'est considérée que dans la mesure où elle est techniquement mesurable.

D'une part, les femmes se retrouvent actuellement à la fois objectivées et dépossédées de certaines dimensions de l'expérience de la procréation et, d'autre part, responsabilisées par rapport aux décisions concernant l'enfant à naître. La problématique liée à l'utilisation 
du diagnostic prénatal est un exemple éloquent de ce que vivent les femmes.

Le diagnostic prénatal peut ainsi conférer à la grossesse son caractère légitime (ou non) et peut même devenir la sanction de l'existence de la grossesse. Au cours d'une recherche menée récemment au Québec, une femme a ainsi témoigné de son attente du résultat de I'amniocentèse :

Les cinq premiers mois, je peux dire que je n'ai pas eu, je n'ai pas vécu ma grossesse. Je n'étais pas capable de voir qu'il y avait un enfant au bout de la course. Je n'étais pas capable de dire : je vais avoir mon bébé à tel mois...

On ne peut prétendre qu'il s'agisse là d'un cas marginal, puisque le même type de considération a été relevé ailleurs (Katz Rothman, 1986). L'existence de la grossesse, donc l'attente de l'enfant à venir, dépend de la sanction médicale et technique de ce que sera l'issue de cette grossesse. Porter un enfant devient porter un enfant répondant à certains critères. Les femmes subissent cette transformation.

Les critères retenus pour définir un enfant " normal " correspondent à des valeurs sociales. C'est socialement qu'est définie l'anormalité. La possibilité technique d'éviter l'anormalité crée des exigences. II s'agit là d'une construction sociale en vertu de laquelle l'accessibilité des moyens plutôt que des choix sociaux explicites oriente les décisions. Puisque l'on peut éviter des enfants anormaux, pourquoi devrions-nous développer des ressources pour leur faciliter l'existence et faciliter leur prise en charge ? Cette option collective, c'est-à-dire éviter la naissance d'enfants portant certains caractères, est alors présentée par la technique comme un " choix " rendu possible aux futures mères, aux futurs parents. C'est ainsi que la responsabilité de concrétiser un choix social est transférée aux seuls individus. Une distinction s'impose cependant ici. Dans un tel cas, il ne s'agit plus d'interrompre une grossesse parce qu'une femme décide de ne pas la poursuivre pour des raisons qui lui appartiennent, comme dans le cas de l'avortement. II s'agit de l'interrompre à partir de l'identification de certains caractères chez l'enfant à naître. Même si des analogies sont possibles puisque dans les deux cas des pressions sociales peuvent intervenir dans la décision, les deux types d'expériences ne doivent pas être confondus.

Cette responsabilisation des femmes à l'égard du " produit de la conception " est souvent abordée sous l'angle d'une amélioration grâce à la technique. Le discours dominant exprime qu'il est maintenant possible d'éviter à des parents d'avoir un enfant porteur d'un handicap grave (notion extensible s'il en est une) et d'éviter la vie à un enfant qui va souffrir. Ce discours doit être analysé pour en dégager la construction : cette " amélioration " tient à une vision technicienne 
de la vie humaine. Cela est d'autant plus important que cette vision peut s'imposer aux femmes qui portent les enfants. C'est d'ailleurs à ce titre qu'elles pourront même faire une "demande " en ce sens. L'existence d'une " demande " de certaines femmes pour un recours à différentes techniques est réelle. L'ancrage social de cette demande est toutefois rarement explicité, alors que c'est en son nom qu'on justifie les développements techniques. Or, ce sont les pressions que les femmes ressentent pour se conformer à leur rôle et assumer leurs responsabilités qui structurent en partie cette demande (De Koninck, 1988).

\section{La déqualification du savoir des femmes}

Le deuxième élément majeur dans la transformation de l'expérience de la reproduction, qui est intrinsèquement lié au premier, est la déqualification du savoir des femmes. Cette déqualification a aussi des fondements qui remontent aux premières techniques. Les propos de De Lee, rapportés plus haut, indiquent comment, dès les premières interventions techniques, on a estimé qu'elles pouvaient se révéler supérieures au processus naturel dans lequel le rôle central dans l'enfantement revient aux femmes.

La déqualification du savoir des femmes s'est toutefois accélérée avec la progression du discours du risque. Le postulat qui sous-tend ce discours veut que la procréation soit un événement risqué. La médicalisation des événements liés à la reproduction a été rendue possible par l'adhésion à ce discours. Or, le lieu ou le siège de ce risque est le corps féminin, ce qui amène son contrôle. Pour éviter le risque inhérent à la procréation, le corps qui fait écran au processus de développement du fœetus doit être surveillé et contrôlé de l'extérieur. Dans la mesure où ce contrôle vient effectivement de l'extérieur et ne fait pas appel à la participation des femmes, celles-ci se voient définitivement reléguées au rang d'objet.

... j'avais l'impression vraiment qu'à un moment donné, j'étais l'enveloppe, le contenant, la personne responsable. Tout dépendait de ce que je pouvais faire, les gens pouvaient avoir des attentes... Contenant qui peut défaillir...

C'est ce discours sur le risque qui justifie le glissement de la femme sujet d'une expérience humaine vers la femme objet d'une expérience technique. La technique n'est pas mise au service de l'expérience féminine et les femmes doivent $s^{\prime}$ en remettre à une expertise extérieure.

Le potentiel reproducteur des femmes en tant que potentiel de vie, capacité et savoir, n'a pas sa place dans le discours du risque. N'y 
trouve pas de place non plus l'expérience de reproduction en tant que siège de la première altérité. Le discours biomédical dominant permet une définition de l'apport des femmes s'éloignant du don de vie pour s'exprimer plutôt en termes d'échelle de risques pour l'enfant.

La mise à jour de l'expérience de la maternité permet de constater que celle-ci est parfois difficile et que malgré une certaine adhésion au discours officiel, l'expérience affective est d'un autre ordre. En mettant l'accent sur les risques, les femmes en arrivent même à développer la conviction qu'elles sont une menace à l'enfant qu'elles portent. Ce phénomène les amène à poser les anomalies potentielles ou effectives de leur futur enfant au sein même de leur propre " défectuosité " ou de leur irresponsabilité, ce qui fait naître ultimement un sentiment de culpabilité renforçant leur supposée inaptitude procréative. Dans la mesure où les femmes ne peuvent plus faire confiance à leur contribution dans la procréation, le pas vers la culpabilisation n'est pas difficile à franchir. C'est dans ce contexte que l'on prétend offrir de nouvelles possibilités de faire des choix.

\section{L'inéluctabilité du recours à la technique}

Ce contexte global d'appréhension des événements liés à la reproduction comme étant à risque, comme devant faire l'objet d'un contrôle serré, ne repose plus seulement sur un souci d'éviter les risques, mais également sur une préoccupation pour la qualité du produit. Ce contexte favorise un développement technique obéissant alors aux lois de sa propre progression. L'inéluctabilité du recours à la technique implique que sa seule existence en rend l'usage impératif. La technique vient donc créer sa propre demande. Ainsi, les femmes responsabilisées, non pas dans leur contribution mais dans l'acceptation des interventions, doivent souscrire aux solutions proposées. Cette attitude de " disponibilité " renforce le caractère inéluctable du recours à la technique. S'il arrive que des femmes réclament ces techniques, c'est qu'elles se sentent responsables et se sentiront coupables si tout ce qui est techniquement possible n'a pas été tenté. Ce sont elles qui portent le risque, ce sont d'autres qui peuvent les contrôler : " au moins, j'aurai tout essayé ».

L'une ou l'autre technique arrive à s'imposer parce qu'elle s'inscrit en continuité logique de la précédente (Ellul, 1977), chaque étape d'un développement annonçant l'étape suivante dans l'imaginaire et éventuellement dans la réalité. Nécessairement parcellaire et axée sur la production, la technique agit directement sur la globalité de l'expérience féminine.

Mais la technique n'intervient pas uniquement sur l'expérience féminine puisque l'expérience de la paternité est également redéfi- 
nie, ramenée progressivement à sa seule dimension biologique. $\mathrm{Ne}$ comportant jamais de certitude biologique, la paternité était jusqu'à tout récemment définie par la voie d'aménagements sociaux. Le père était en fait le père social. Ce qu'apporte la technique à la paternité, ce n'est pas un enrichissement de sa dimension psychosociale, mais bien plutôt une certaine réduction vers la biologie.

Cette tendance est d'autant plus paradoxale que, dans le cadre d'une certaine évolution des rapports sociaux de sexe, c'est plutôt vers la complexification de la paternité sociale, en élargissant les rôles et les responsabilités qui lui sont rattachées, que l'on prétend vouloir tendre. En effet, on a souvent considéré que le rôle des hommes, limité à celui de pourvoyeur, était réducteur et qu'il fallait l'enrichir par la reconnaissance de la part à prendre dans l'ensemble des responsabilités parentales. Selon cette vision des choses, la dimension psychosociale de la parentalité, soit celle de la relation avec l'enfant, occupe alors un certain espace. Or, le processus actuel de biologisation de la paternité, à savoir que le rôle de géniteur devient le rôle dominant pour définir la paternité (ramenant celle-ci aux spermatozoïdes), s'inscrit en toute cohérence avec une logique technique ne pouvant que mesurer et contrôler le manipulable.

\section{Que voulons-nous pour notre société ?}

Face aux techno-sciences, l'éthique est fragile puisqu'elle n'est pas de l'ordre des moyens mais qu'elle détermine une finalité, soit une vision globale d'une activité. Elle pose des limites en affirmant des principes et des valeurs ${ }^{2}$. Les techno-sciences sont sans limites dans leur développement : elles planifient la succession des démarches, c'est-à-dire les moyens. Sans vouloir articuler ici le rapport difficile entre l'éthique et les techno-sciences - ce qui dépasserait le cadre de cet exposé - , on peut dire avec Ladrière que « les valeurs éthiques jouent un rôle vraiment central dans le système de valeurs d'une culture, car ce sont elles qui commandent les normes de l'action et donc déterminent en définitive les modèles de comportement, les principes de choix, les critères d'appréciation et les motivations à partir desquelles sont fixés les objectifs concrets à court ou à long terme » (Ladrière, $1979:$ 137). Bref, on ne peut échapper à l'éthique dans la mesure où elle contribue, de façon déterminante, à structurer nos rapports sociaux et notre vie quotidienne en société. Une des difficultés majeures de l'éthique dans le rapport aux techno-sciences, c'est d'affirmer des normes et des valeurs socialement choisies qui puissent concrètement orienter les techniques, et même les arrêter si celles-ci ne deviennent que pouvoir sur l'humain et négation de sa liberté fondamentale. 
La démarche que nous proposons n'est que l'esquisse d'une articulation possible entre l'éthique et le développement des technosciences en matière de procréation humaine. Nous proposons des fins (des valeurs) à partir desquelles notre société fonctionne et qui peuvent être socialement affirmées et des moyens (des normes) qui nous permettent d'orienter les techniques, voire de suspendre leur développement si elles mettent en péril des valeurs jugées socialement fondamentales.

\section{Les valeurs de la maternité et de la parentalité}

Notre société québécoise et canadienne est pluraliste. Mais ce pluralisme des valeurs n'empêche pas un consensus autour de certaines valeurs ou principes jugés, sur le plan social, comme le fondement même de notre société. Ces valeurs et principes permettent de poser des balises à nos conduites sociales et de prendre des décisions sociales ayant des conséquences sur l'avenir. Les techniques de procréation humaine constituent un domaine où il importe d'affirmer certaines valeurs car se joue, à travers ces techno-sciences, notre avenir collectif. L'utilisation de celles-ci intervient directement dans les expériences de maternité et de paternité, expériences de parentalité qui sont au fondement de notre vie sociale. Une société sans enfants est une société sans lendemain : les enfants sont essentiels à la survie d'une société.

Il est urgent de réfléchir au sens que nous donnons à la fonction de procréation. C'est par cet exercice que l'on arrive à dégager les valeurs auxquelles nous tenons et qui donnent sens. Les premières valeurs que nous suggérons ici sont celles de la maternité et de la parentalité.

La définition techno-scientifique de l'expérience de la maternité occulte l'expérience humaine qui est d'abord la relation à l'autre. $C^{\prime}$ est dans la relation à l'autre que réside le sens de la maternité. Devenir mère est d'abord une expérience d'altérité. Ce qui enrichit cette expérience est la conscience de l'autre en tant que distinct de soi, autre issu de soi et à qui l'on donne la vie. Autre qui construira son identité en dehors de soi alors que c'est en soi qu'il aura trouvé son origine. Ramener l'expérience de la maternité à ses seules dimensions physiques la vide de son sens.

De la même façon, on peut prétendre que c'est en mettant l'accent sur la dimension psychosociale de la paternité qu'on respecte le sens qui peut lui être conféré. Distincte de l'expérience de la maternité, puisqu'elle ne prend pas ses racines dans le même type de processus, la paternité est également d'abord une relation à l'autre et c'est sur le développement de cette relation qu'il importe de mettre 
l'accent. Cette valeur globale, soit la dimension humaine de la parentalité, ne se réduit pas à une parentalité biologique mais plutôt s'élargit à la relation parent-enfant.

\section{Les droits fondamentaux de la personne humaine}

Notre société est fondée sur la démocratie qui, comme structure politique, repose elle-même sur des valeurs dont la Charte des droits et libertés de la personne témoigne. Ce qui sous-tend la Charte, c'est la philosophie des droits de la personne ${ }^{3}$. Reconnaître l'autre comme personne, c'est lui reconnaître les mêmes droits qu'à soi. C'est fondamentalement reconnaître en lui ou elle la dignité d'humain ${ }^{4}$. C'est ne pas le traiter simplement comme un moyen mais comme une fin en soi.

La dignité humaine se reconnaît au-delà des différences de sexes, de couleurs, d'intelligence ou de fortune. "Égaux en droits ", dans l'énoncé même de ce principe, on présuppose des inégalités naturelles ou sociales qui sont compensées par un régime de droit permettant de les dépasser. Au cœur de la philosophie des droits de la personne repose donc la notion d'altérité et de respect de la différence. Ce respect de la différence et de la dignité d'autrui permet d'affirmer les droits de la personne humaine comme une référence éthique importante dans le débat sur les technologies de procréation humaine. Notre société reconnaît dans l'autre l'altérité, la différence et va protéger, par des règles de droit, ces différences. Les techniques de procréation humaine, en instrumentalisant l'enfant et la femme, en standardisant le produit, en rejetant " l'anormal ", ne font que s'éloigner de la philosophie des droits de la personne sur laquelle repose notre société.

\section{Dire la norme à travers la techno-science}

Comment ces valeurs abstraites, et qui pourtant sont au cœur de nos institutions et de notre morale sociale, peuvent-elles inspirer tangiblement la régulation du développement techno-scientifique en matière de procréation humaine ?

Bref, comment définir la norme acceptable pour les techno-sciences dans une démocratie comme la nôtre ?

La fin de cet exposé n'est pas d'établir si la congélation des embryons est acceptable ou non, ni de définir le cadre dans lequel le diagnostic prénatal doit se pratiquer ou être accessible. Nous proposons des axes de réflexion, des questions et des pistes qui permettraient de penser de façon globale mais précise le développement de techno-sciences dans le domaine de la procréation humaine. 
Penser le développement des techno-sciences, c'est s'interroger sur la finalité de celles-ci. Pourquoi cherchons-nous à contrôler de plus en plus le développement de l'embryon et du fœetus ? Quel est le but ultime du diagnostic prénatal si ce n'est de définir à l'avance et de façon de plus en plus précise ce que sera l'enfant à venir ? Quelle incidence tout cela aura-t-il sur l'expérience de la maternité et de la parentalité si les techno-sciences en viennent à définir ce qu'elles doivent être?

Vient alors la question des limites. On peut s'interroger, tout d'abord, sur la légitimité d'un développement non contrôlé des techno-sciences dans le domaine si fondamental de la procréation humaine. II n'est pas question de tout rejeter en bloc, mais plutôt de s'interroger sur les limites de l'application de ces techno-sciences en regard des finalités poursuivies. Où conduit la recherche d'un contrôle plus important sur la procréation et son déroulement ? Qui doit décider de l'orientation de notre société et par quel mécanisme?

Ces interrogations sur les finalités et les limites du développement techno-scientifique en matière de procréation humaine nous obligent à penser l'éthique non plus en termes d'éthique individuelle (penser en fonction du couple ou de la personne qui demande) mais en raisonnant à partir d'une éthique collective fondée sur les valeurs qui soudent notre société démocratique et que nous avons évoquées plus haut. Ces considérations nous amènent à formuler, en conclusion de ce travail, deux recommandations.

\section{A. Le décret d'un moratoire sur les recherches en procréation humaine}

Les enjeux des technologies de procréation humaine sont importants, nous en avons soulevé quelques-uns. Le développement de ces technologies a été extrêmement rapide comme s'il y avait urgence d'agir et une priorité telle qu'on devait faire fi de toutes autres considérations. Il serait temps de s'arrêter afin de s'accorder des moments de réflexion pour définir les finalités et les limites du développement de ces techniques.

L'idée d'un moratoire, c'est-à-dire d'un freinage provisoire du progrès scientifique et technique, relève en effet du simple principe de prudence. Personne ne serait assez fou pour se lancer à toute allure au volant d'une voiture puissante sans s'être assuré que les freins marchent. Le seul moyen de s'en assurer c'est de freiner effectivement tant qu'on ne va pas trop vite. Nous qui sommes engagés dans un processus de changement accéléré, sommes exactement dans la même situation. Or, nous prétendons qu'il est inutile d'essayer de freiner, puisqu'en principe (principe qu'aucune expérience n'a jamais pu vérifier) le progrès accroît la souveraineté de l'homme 
et sa maîtrise sur les choses, lui-même et la société. C'est le type même de comportement imprudent qui consiste à ne pas s'assurer que nous avons les moyens de stabiliser une situation évoluant rapidement au cas où les choses tournent mal... Puisque l'homme est un être imparfait voué à vivre dans un monde fini, l'accroissement indéfini de la puissance offerte aux individus et aux groupes ne peut qu'entrer tôt ou tard en contradiction avec les limites spatio-temporelles de l'expérience humaine et détruire les cadres naturels et sociaux de la vie humaine. On peut bien sûr rétorquer que les limites ne sont pas aujourd'hui atteintes. Est-ce sûr ? De toutes façons (sic), il paraît difficile de nier que, tôt ou tard, elles le seront. Si elles ne sont pas choisies elles risquent de s'imposer d'elles-mêmes de manière fort déplaisante. Ce qui ne paraît pas souhaitable et milite en faveur de l'autolimitation préventive (Cerezuelle, 1988 : 114-115).

\section{B. La réalisation de recherches sur les mécanismes de} développement des techno-sciences et sur les impacts psychosociaux de ces développements technologiques en matière de procréation humaine

La logique qui domine le développement des techno-sciences sert les lois de l'économie de marché. Cela implique que son développement, dans la mesure où il peut correspondre à un développement économique, ne connaît pas de limites, si ce ne sont les limites sociales qu'on peut lui imposer. Au nom de notre avenir collectif, des analyses sur les mécanismes qui orientent les prises de décision dans le domaine de la procréation humaine doivent donc être complétées. De telles analyses devraient permettre de prendre une distance avec la logique technique pour en dégager l'articulation avec les lois de l'économie de marché, alors que la réalité à laquelle s'adresse le domaine de la procréation est une réalité dont les composantes psychosociales jouent un rôle déterminant. La procréation n'est pas un bien commercial et il s'impose de saisir les éléments qui favorisent son traitement selon cette approche.

Nos connaissances sur la réalité humaine en cause dans les développements technologiques en procréation assistée n'ont aucune commune mesure avec les connaissances technologiques. II importe et il est même urgent de développer ce type de connaissances.

Des recherches qualitatives donnant la parole à la population et permettant d'appréhender l'expérience vécue dans le domaine de la procréation humaine devraient devenir une priorité. Ces recherches auraient comme objectif, notamment, d'approfondir la " demande " et le recours aux technologies. C'est en effet au nom de la demande sociale que l'on justifie les recherches et l'utilisation des techniques. Mais qu'en est-il de cette demande ? Qu'en savons-nous réellement ? 
Tant que des recherches qualitatives permettant d'étayer la construction de cette demande ne sont pas menées à terme, nous ne pouvons continuer à prétendre qu'elle justifie les investissements actuels selon l'orientation qu'on leur donne.

En conclusion, le défi pour penser le développement des technosciences en matière de procréation humaine est de les voir globalement, dans l'ensemble de leurs effets et conséquences au regard des principes au fondement de notre société. C'est-à-dire penser la techno-science comme au service d'une vision du monde où l'expérience humaine est respectée (reconnaissance de la différence de l'autre, reconnaissance de la complémentarité de l'expérience de la maternité et de la paternité). Enfin, il s'agit d'affirmer que la procréation est d'abord une expérience et une aventure humaine et, pour paraphraser Albert Jacquart, "un éloge de la différence ".

\section{Notes}

${ }^{1}$ " One may well ask himself whether the brief and moderate compression of the head in a skillfully performed forceps operation is not less dangerous to the integrity of the brain than the prolonged pounding and congestion it suffers from a hard spontaneous delivery? ...should we anticipate the dangers and, as a routine, make the first stage of labor less painful and shorter and eliminate the second stage by a surgical delivery? " (Jos. B. De Lee, "The Prophylactic Forceps Operation ", The American Journal of Obstetrics and Gynecology, 1920 : 42).

${ }^{2}$ " L'éthique est une valeur que l'on attache à tous les acteurs sans exception et à toutes les activités humaines, y compris par conséquent l'activité technique, suivant qu'on l'accomplit correctement ou non, honnêtement ou non, avec dignité ou non." (J. Freund, $1983: 32$ ).

${ }^{3}$ "Peut-on soutenir que [...] il existe un accord des esprits sur la nécessité de préserver, comme valeur fondamentale, la dignité de la personne, le caractère sacré de l'individu ? Si c'était effectivement le cas, nous disposerions là d'un fondement accepté des droits de l'homme : en effet, une fois adoptée comme valeur première la protection de l'individu, les conditions de son épanouissement, bref les droits de l'homme, en découlent tout naturellement comme une exigence catégorique. " (Guy Haarscher, Philosophie des droits de I'homme, $1987:$ 107).

II ne peut s'agir dans le cadre de ce texte de reprendre la philosophie des droits de la personne dans sa complexité et ses ambiguïtés; cependant dans notre démarche nous nous appuyons sur les propos développés par $\mathrm{G}$. Haarscher.

${ }^{4}$ On pourra se référer au chapitre quatre du livre de Janos Kis, L'égale dignité. Essai sur les fondements des droits de l'homme, 1989. 


\section{Références bibliographiques}

Actes du colloque Génétique, Procréation et Droit (1985). Paris : Actes Sud.

ARDITTI, Rita et al. (eds.) (1984). Test-Tube Women : What Future For Motherhood? Londres : Pandora Press.

ARneY, William Ray (1982). Power and The Profession of Obstetrics. Chicago : The University of Chicago Press.

BAUdoIn, Jean-Louis et Catherine LABrusSe-Riou (1987). Produire l'homme : de quel droit ? Étude juridique et éthique des procréations artificielles. Paris : Presses Universitaires de France.

BAYles, Michael D (1984). Reproductive Ethics. Englewood Cliffs, NJ : PrenticeHall Inc.

Cerezuelle, Daniel (1988). "Réflexions sur l'autonomie de la technique », dans Évaluer la technique, G. Hottois (éd.). Paris : Vrin, p. 114-115.

Conen, Sherrill et Nadine TAub (eds.) (1989). Reproductive Laws for the 1990s. Clifton, NJ : Humana Press.

Collectif : Sortir la maternité du laboratoire, (1988). Actes du colloque international organisé par le Conseil du statut de la femme et tenu à Montréal les 29, 30 et 31 octobre 1987. Québec : Bibliothèque nationale du Québec, Gouvernement du Québec.

COREA, Gena (1985). The Mother Machine. Reproductive Technologies from Artificial Insemination to Artificial Wombs. New York : Harper \& Row.

COREA, Gena et al. (1985). Man-Made Women : How New Reproductive Technologies Affect Women. Londres : Hutchinson.

DE KonINCK, Maria (1988). Femmes, enfantement et changement social : Le cas de la césarienne. Thèse de doctorat en sociologie. Québec : Université Laval.

DelaisI de Parseval, Geneviève et A. Janaud (1983). L'Enfant à tout prix. Paris : Éditions du Seuil/Actuel.

De LeE, Jos. B. (1920). "The Prophylactic Forceps Operation ", The American Journal of Obstetrics and Gynecology. 1 : 34-44.

Eltul, Jacques (1977). Le système technicien. Paris : Calman-Lévy.

EluuL, Jacques (1983). " Recherche pour une éthique dans une société technicienne ", dans Éthique et technique, Annales de l'Institut de philosophie et de sciences morales. Bruxelles : Éditions de l'Université Libre de Bruxelles.

Fagot-Largeau, Anne et Geneviève Delaisi de parseval (1987). "Les droits de l'embryon (fœetus) humain, et la notion de personne humaine potentielle ", Revue de Métaphysique et de Morale, $\mathrm{n}^{\circ} 3$.

FREUND, J. (1983). " Observations sur la finalité respective de la technique et de l'éthique ", dans Éthique et technique, Annales de I'Institut de philosophie et de sciences morales. Bruxelles : Éditions de l'Université Libre de Bruxelles.

Gouvernement du QuéBEC, ministère de la Santé et des Services sociaux (1988). Rapport du comité de travail sur les nouvelles technologies de reproduction humaine. Québec : Gouvernement du Québec.

HAARSCher, Guy (1987). Philosophie des droits de l'homme. Bruxelles : Éditions de I'Université Libre de Bruxelles. 
HuLl, Richard T. (éd.) (1990). Ethical Issues in the New Reproductive Technologies.

Katz Rothman, Barbara (1986). The Tentative Pregnancy : Prenatal Diagnosis and the Future of Motherhood. New York : Viking Penguin Books.

Katz Rothman, Barbara (1989). Recreating Motherhood: Ideology and Technology in a Patriarcal Society. New York : W.W. Norton and Company. Belmont, CA : Wadsworth Publishing Company.

KLEIN, Renate D. (1989). Infertility : Women Speak Out About Their Experiences of Reproductive Medicine. Londres : Pandora Press.

KIS, Janos (1989). L'égale dignité. Essai sur les fondements des droits de l'homme. Paris : Seuil, coll. Esprit.

KNOPPERS, Bartha Maria (1986). Conception artificielle et responsabilité médicale. Cowansville : Éditions Yvon Blais.

LADRIÈRE, Jean (1979). Les enjeux de la rationalité. Paris : Aubier-Montaigne/ Unesco.

OAKLEY, Ann (1984). The Captured Womb. Oxford : Basic Blackwell.

O'BRIEN, Mary (1987). La dialectique de la reproduction. Montréal : Les Éditions du remue-ménage.

OVERALL, Christine (ed.) (1989). The Future of Human Reproduction. Toronto : Women's Press.

OVERALL, Christine (1987). Ethics and Human Reproduction : A Feminist Analysis. Boston : Allen and Unwin.

$\mathrm{RICH}$, Adrienne (1976). Of Woman Born : Motherhood as Experience and Institution. New York : W.W. Norton.

SINDING, Christiane (1984). "Le gène, la norme et le pathologique ", Revue Après-Demain, $\mathrm{n}^{\circ}$ 266; juillet-sept. : 28-30.

Spallone, Patricia et Deborah Lynn SteinBerG (eds.) (1987). Made to Order: The Myth of Reproductive and Genetic Progress. Oxford, New York and Toronto : Pergamon Press.

STANWORTH, Michelle (ed.) (1987). Reproductive Technologies : Gender, Motherhood and Medicine. Minneapolis : University of Minnesota Press.

Thuiluier, Pierre (1981). Darwin \& Co. Bruxelles : Éditions Complexe.

WARNOCK, Mary (1985). A Question of Life : The Warnock Report on Human Fertilisation and Embryology. New York : Basic Blackwell Inc.

WERTZ, Richard W. et Dorothy C. WERTZ (1979). Lying-In, A History of Childbirth in America. New York : Schocken Books.

Whiteford, Linda M. et Marylin L. Poland (eds.) (1989). New Approaches to Human Reproduction : Social and Ethical Dimensions. Boulder, CO : Westview Press. 